\title{
Photoneutron Reaction Data for Nuclear Physics and Astrophysics
}

\author{
Hiroaki Utsunomiya ${ }^{1, *}$, Therese Renstrøm ${ }^{2}$, Gry Merete Tveten ${ }^{2}$, Ioana Gheorghe ${ }^{3}$, Dan Mihai Filipescu ${ }^{3}$, Sergey \\ Belyshev ${ }^{4}$, Konstantin Stopani ${ }^{5}$, Hongwei Wang ${ }^{6}$, Gongtao Fan ${ }^{6}$, Yiu-Wing Lui ${ }^{7}$, Dmytro Symochko ${ }^{8}$, Stephane Goriely ${ }^{9}$, \\ Ann-Cecilie Larsen ${ }^{2}$, Sunniva Siem ${ }^{2}$, Vladimir Varlamov ${ }^{5}$, Boris Ishkhanov ${ }^{5}$, Tudor Glodariu ${ }^{3}$, Mateusz Krzysiek ${ }^{3,10}$, Daiki \\ Takenaka $^{1}$, Takashi Ari-izumi ${ }^{1}$, Sho Amano ${ }^{11}$, Shuji Miyamoto ${ }^{11}$ \\ ${ }^{1}$ Department of Physics, Konan University, 658-8501 Kobe, Japan \\ ${ }^{2}$ Department of Physics, University of Oslo, N-0316, Oslo, Norway \\ ${ }^{3}$ ELI-NP, "Horia Hulubei" National Institute for Physics and Nuclear Engineering (IFIN-HH) , 077125 Bucharest-Magurele, Romania \\ ${ }^{4}$ Department of Physics, Lomonosov Moscow State University, Moscow 119991, Russia \\ ${ }^{5}$ Skobeltsyn Instutute of Nuclear Physics, Lomonosov Moscow State University, Moscow 119991, Russia \\ ${ }^{6}$ Shanghai Institute of Applied Physics, Shanghai, 201800, China \\ ${ }^{7}$ Cyclotron Institute, Texas A\&M University, Texas 77843, USA \\ ${ }^{8}$ Technische Universität Darmstadt, 64289 Darmstadt, Germany \\ ${ }^{9}$ Institutd'Astronomie et d'Astrophysique, Université Libre de Bruxelles, 1050 Brussels, Belgium \\ ${ }^{10}$ Institute of Nuclear Physics Polish Academy of Sciences, PL-31342 Krakow, Poland \\ ${ }^{11}$ Laboratory of Advanced Science and Technology for Industry, University of Hyogo, Hyogo 678-1205, Japan
}

\begin{abstract}
We discuss the role of photoneutron reaction data in nuclear physics and astrophysics in conjunction with the Coordinated Research Project of the International Atomic Energy Agency with the code F41032 (IAEA-CRP F41032).
\end{abstract}

\section{PHOENIX Collaboration for the IAEA- CRP F41032}

The Coordinated Research Project of the International Atomic Energy Agency with the code F41032 (IAEACRP F41032) was launched in 2016 as a 5-years** project. The goal of the project is to publish two compilations of an updated photonuclear data library and a reference database of photon strength functions in 2020.

We have established an experimental collaboration called the PHOENIX (PHOto-Excitation and Neutron emIssion cross $[\mathrm{X}]$ sections) Collaboration for the IAEA-CRP in the $\gamma$-ray beam line GACKO (GAmma Collaboration hutch of KOnan university) of the NewSUBARU synchrotron radiation facility in Japan [1] The PHOENIX Collaboration is hosted by the Konan University; the collaboration involves the University of Oslo, ELI-NP (Extreme Light Infrastructure - Nuclear Physics), Moscow State University, Shanghai Institute of Applied Physics, Texas A\&M University, Technische Universität Darmstadt, Université Libre de Bruxelles, and University of Hyogo.

In this collaboration, we are measuring $(\gamma, \mathrm{xn})$ cross sections with $\mathrm{x}=1-4$ for the first compilation (photonuclear data) and $(\gamma, 1 \mathrm{n})$ cross sections near oneneutron threshold for the second compilation (photon

\footnotetext{
*Corresponding author: hiro@konan-u.ac.jp

** The data acquisition is limited to 3 years for data evaluations and model calculations.
}

strength functions). A list of nuclei to be measured in the PHOENIX Collaboration is given in Table 1.

Table 1. Nuclei covered by the PHOENIX Collaboration.

\begin{tabular}{|c|c|c|}
\hline & Photonuclear data & $\begin{array}{l}\text { Photon strength } \\
\text { functions }\end{array}$ \\
\hline 2015 & ${ }^{209} \mathrm{Bi},{ }^{9} \mathrm{Be}$ & ${ }^{205} \mathrm{Tl},{ }^{203} \mathrm{Tl},{ }^{89} \mathrm{Y}$ \\
\hline 2016 & ${ }^{197} \mathrm{Au},{ }^{169} \mathrm{Tm},{ }^{89} \mathrm{Y}$ & $\begin{array}{c}{ }^{192} \mathrm{Os},{ }^{185} \mathrm{Re},{ }^{138} \mathrm{Ba}, \\
{ }^{137} \mathrm{Ba},{ }^{64} \mathrm{Ni},{ }^{61} \mathrm{Ni},{ }^{60} \mathrm{Ni} \\
{ }^{58} \mathrm{Ni},{ }^{13} \mathrm{C}\end{array}$ \\
\hline 2017 & ${ }^{181} \mathrm{Ta},{ }^{165} \mathrm{Ho},{ }^{59} \mathrm{Co}$ & 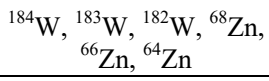 \\
\hline 2018 & ${ }^{159} \mathrm{~Tb},{ }^{139} \mathrm{La},{ }^{103} \mathrm{Rh}$ & ${ }^{160} \mathrm{Gd},{ }^{158} \mathrm{Gd},{ }^{156} \mathrm{Gd} \mathrm{Gd}$, \\
\hline
\end{tabular}

We provide $(\gamma, \mathrm{xn})$ cross section data to resolve the long-standing discrepancy between the Livermore and Saclay data of partial photoneutron cross sections. In many cases, Saclay provided larger cross sections for $(\gamma, 1 \mathrm{n})$ reactions than Livermore and vice versa for $(\gamma, 2 \mathrm{n})$ reactions [2]. The ratio of the Saclay data to the Livermore data of integrated total cross sections, $R^{\text {int }}=\sigma_{S}^{\text {int }} / \sigma_{L}^{\text {int }}$, varies from 0.65 to 1.35 over 19 nuclei from ${ }^{51} \mathrm{~V}$ to ${ }^{238} \mathrm{U}$. As shown in Fig. 1, the ratio for 
integrated partial cross sections averaged over the 19 nuclei is very different; it is $\left\langle R^{\text {int }}(1 n)\right\rangle=1.07$ and $<R^{\text {int }}(2 n)>=0.84$ for the $(\gamma, 1 \mathrm{n})$ and $(\gamma, 2 \mathrm{n})$ reactions, respectively [3].

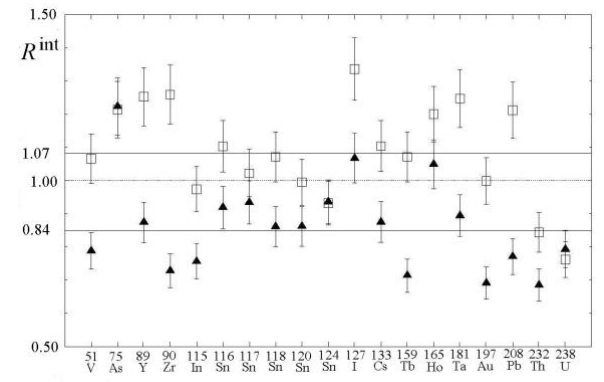

Fig. 1 The ratio of the Saclay data to the Livermore data of integrated cross sections, $R^{\text {int }}=\sigma_{S}^{\text {int }} / \sigma_{L}^{\text {int }}$, for $(\gamma, 1 \mathrm{n})$ (open squares) and $(\gamma, 2 \mathrm{n})$ (solid triangles) reactions.

We also provide $(\gamma, 1 \mathrm{n})$ cross section for specific nuclei near the one-neutron threshold for an absolute normalization of the photon strength function below neutron threshold obtained with the Oslo method [4]. Figure 2 shows the resulting photon strength function for ${ }^{117} \mathrm{Sn}$ constructed experimentally, showing the presence of an extra strength over the neuron threshold at 6.94 $\mathrm{MeV}$ that is attributed to a pygmy E1 resonance [5].

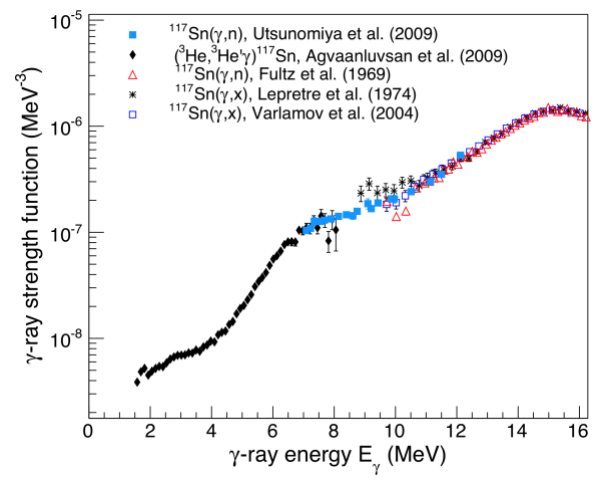

Fig. 2 (color online) Photon strength function for ${ }^{117} \mathrm{Sn}$ constructed from the photoneutron data and the data of the Oslo method.

We also provide $(\gamma, 1 \mathrm{n})$ cross sections systematically over an isotopic chain to reach a unified understanding of $(\gamma, n)$ and $(n, \gamma)$ cross sections with the $\gamma$-ray strength function method [6,7]. This method effectively deduces $(\mathrm{n}, \gamma)$ cross sections for radioactive nuclei involved in the isotopic chains as a result of this unified and comprehensive approach.

The PHOENIX Collaboration will continue after the IAEA-CRP. Nuclei for which $(\gamma, \mathrm{xn})$ cross sections will be measured after 2018 may include ${ }^{112} \mathrm{Sn},{ }^{116} \mathrm{Sn},{ }^{120} \mathrm{Sn}$, ${ }^{124} \mathrm{Sn},{ }^{208} \mathrm{~Pb},{ }^{232} \mathrm{Th}$, and ${ }^{238} \mathrm{U}$.

\section{Direct Neutron-Multiplicity Sorting with a Flat-Response Detector}

We have long used a high-efficiency neutron detector of Livermore type to measure $(\gamma, 1 \mathrm{n})$ cross sections near the one-neutron threshold. This detector consisting of three rings of total twenty ${ }^{3} \mathrm{He}$ proportional counters embedded in a polyethylene moderator has a strong dependence of the detection efficiency on neutron kinetic energies. We use the so-called ring-ratio (RR) technique developed at Livermore [8] to determine the mean kinetic energies of neutrons. However, because of the RR technique, we limited ourselves to $(\gamma, 1 \mathrm{n})$ cross section measurements below the two-neutron threshold, where the number of detected neutrons is equal to the number of $(\gamma, 1 \mathrm{n})$ reactions. In the one-neutron channel, the ring ratio can surely determine the mean kinetic energies of neutrons produced in the $(\gamma, 1 n)$ reaction. However, when we go above the two-neutron threshold, we immediately encounter an essential problem with the $\mathrm{RR}$ technique because there is no way to know in what reaction neutrons are produced and detected.

Let us consider measurements of $(\gamma, \mathrm{xn})$ cross sections with neutron-multiplicity $\mathrm{x}=1-3$ with a neutron detector of Livermore type by using a pulsed $\gamma$-ray beam. The frequency of the pulsed beam is typically $1 \mathrm{~ms}$ because of the long neutron-moderation time of the polyethylene. During the $1 \mathrm{~ms}$ time-interval between two successive $\gamma$ ray pulses, we detect neutrons. According to the number of detected neutrons $(n)$, let us name neutron events as single $\left(N_{s}\right)$, double $\left(N_{d}\right)$, and triple $\left(N_{t}\right)$ events corresponding to $n=1,2$, and 3 , respectively. The neutron events are expressed as follows.

$$
\begin{aligned}
& N_{s}=N_{1} \cdot \varepsilon\left(E_{1}\right)+ \\
& N_{2} \cdot \varepsilon\left(E_{21}\right) \cdot\left(1-\varepsilon\left(E_{22}\right)\right)+ \\
& N_{2} \cdot \varepsilon\left(E_{22}\right) \cdot\left(1-\varepsilon\left(E_{21}\right)\right)+ \\
& N_{3} \cdot \varepsilon\left(E_{31}\right) \cdot\left(1-\varepsilon\left(E_{32}\right)\right) \cdot\left(1-\varepsilon\left(E_{33}\right)\right)+ \\
& N_{3} \cdot \varepsilon\left(E_{32}\right) \cdot\left(1-\varepsilon\left(E_{31}\right)\right) \cdot\left(1-\varepsilon\left(E_{33}\right)\right)+ \\
& N_{3} \cdot \varepsilon\left(E_{33}\right)\left(1-\varepsilon\left(E_{31}\right)\right)\left(1-\varepsilon\left(E_{32}\right)\right) \\
& N_{d}=N_{2} \cdot \varepsilon\left(E_{21}\right)^{2}+ \\
& N_{3} \cdot \varepsilon\left(E_{31}\right) \cdot \varepsilon\left(E_{32}\right) \cdot\left(1-\varepsilon\left(E_{33}\right)\right)+ \\
& N_{3} \cdot \varepsilon\left(E_{31}\right) \cdot \varepsilon\left(E_{33}\right) \cdot\left(1-\varepsilon\left(E_{32}\right)\right)+ \\
& N_{3} \cdot \varepsilon\left(E_{32}\right) \cdot \varepsilon\left(E_{33}\right) \cdot\left(1-\varepsilon\left(E_{31}\right)\right) \\
& N_{t}=N_{3} \cdot \varepsilon\left(E_{31}\right) \cdot \varepsilon\left(E_{32}\right) \cdot \varepsilon\left(E_{33}\right)
\end{aligned}
$$

Here $N_{x}(\mathrm{x}=1,2,3)$ is the number of $(\gamma, \mathrm{xn})$ reactions induced by $\gamma$-ray pulses and $E_{i j}$ is the kinetic energy of the $j$-th neutron emitted in the $(\gamma, i$ n) reaction. Note that the neutron detection efficiency $(\varepsilon)$ depends on the neutron kinetic energy.

In the triple neutron event, obviously all of the first-, second-, and third-emitting neutrons are detected when the $(\gamma, 3 \mathrm{n})$ reaction takes place. The second term of the 
double neutron event means that the first- and secondemitting neutrons are detected, while the third-emitting neutron is undetected when the $(\gamma, 3 n)$ reaction takes place. The single neutron event consists of six terms in which the third term means that the second-emitting neutron is detected, while the first-emitting neutron is undetected when the $(\gamma, 2 \mathrm{n})$ reaction takes place. Note that kinetic energy of the $j$-th emitting neutron is, in principle, different from that of the $k$-th $(k \neq j)$ emitting neutron. Thus, the RR technique, which is applied to the experimental quantities, $N_{s}, N_{d}$, and $N_{t}$, does not work to determine neutron kinetic energies.

One can immediately see that the only solution to determine the number of $(\gamma, \mathrm{xn})$ reactions is to make the neutron detection efficiency $(\varepsilon)$ independent of neutron kinetic energies. In this case, the neutron events are expressed as follows.

$$
\begin{aligned}
& N_{s}=N_{1} \cdot \varepsilon+N_{{ }_{2}} C_{1} \cdot \varepsilon \cdot(1-\varepsilon)+ \\
& N_{3{ }_{3}} C_{1} \cdot \varepsilon \cdot(1-\varepsilon)^{2} \\
& N_{d}=N_{2} \cdot \varepsilon^{2}+N_{3_{3}} C_{2} \cdot \varepsilon^{2} \cdot(1-\varepsilon) \\
& N_{t}=N_{3} \cdot \varepsilon^{3}
\end{aligned}
$$

Now one can solve a set of Eqs. (4), (5), and (6) to determine $N_{1}, N_{2}$, and $N_{3}$, the numbers of $(\gamma, \mathrm{xn})$ reactions $(\mathrm{x}=1,2,3)$ induced by the $\gamma$-ray beam from the experimental quantities, $N_{s}, N_{d}$, and $N_{t}$. Note that $\varepsilon$ is now a constant. It is straightforward to determine $(\gamma, \mathrm{xn})$ cross sections $\left(\sigma_{x n}\right)$ by $\sigma_{x n}=N_{x} /\left(N_{\gamma} \cdot N_{T}\right)$ with the number of $\gamma$-rays $\left(N_{\gamma}\right)$ incident on target nuclei $\left(N_{T}\right)$ per unit area.

We have developed a flat-response neutron detector (FRD) for $(\gamma, x n)$ cross section measurements in the IAEA-CRP [9]. The detector consists of three concentric rings of 4, 9, and $18{ }^{3} \mathrm{He}$ counters embedded in a $46 \mathrm{~cm}$ (horizontally) x $46 \mathrm{~cm}$ (vertically) x $50 \mathrm{~cm}$ (along the beam axis) polyethylene moderator at the distances of $5.5,13.0$ and $16.0 \mathrm{~cm}$ from the $\gamma$-ray beam axis, respectively. Figure 3 shows a photograph of the flatresponse detector.

The total and partial detection efficiencies of the FRD are shown in Fig. 4 along with results of the calibration with a ${ }^{252} \mathrm{Cf}$ source and GEANT4 simulation.

The emission rate of the ${ }^{252} \mathrm{Cf}$ source was calibrated at the National Metrology Institute of Japan. Results are plotted at the average energy $(3 T / 2)$ of the ${ }^{252} \mathrm{Cf}$ neutron spectrum corresponding to the temperature $T=1.42 \mathrm{MeV}$ [10], where the fission spectrum is given by the Maxwell-Boltzmann distribution, $P(E) \propto E^{1 / 2}$. $\exp (-E / T)$. The GEANT4 simulation was performed for monochromatic neutrons (dotted lines) and neutronevaporation spectra (solid lines) expressed by $P(E) \propto E$ - $\exp (-E / T)$ with the Weisskopf-Ewing model [11]. Results for the evaporation spectra are shown at the average neutron energy $(2 T)$. The total efficiency for the evaporation spectra average over the energy range of $0.01-5.0 \mathrm{MeV}$ is $36.5 \%$ with one standard deviation of $1.6 \%$. The neutron energy range was investigated for ${ }^{209} \mathrm{Bi}(\gamma, \mathrm{xn})$ reactions at $40 \mathrm{MeV}$ by means of a Monte Carlo statistical model calculation [12]. The calculation shows that the mean neutron energy for the $(\gamma, \mathrm{xn})$ channel with $x=2-5$ lies within the energy range.

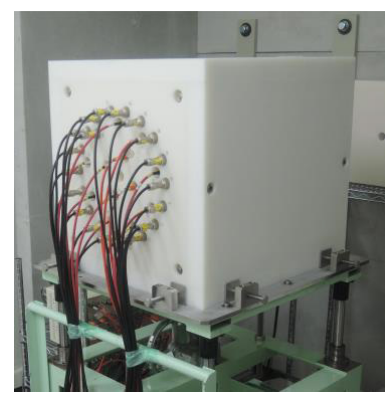

Fig. 3 (color online) Flat-response neutron detector.

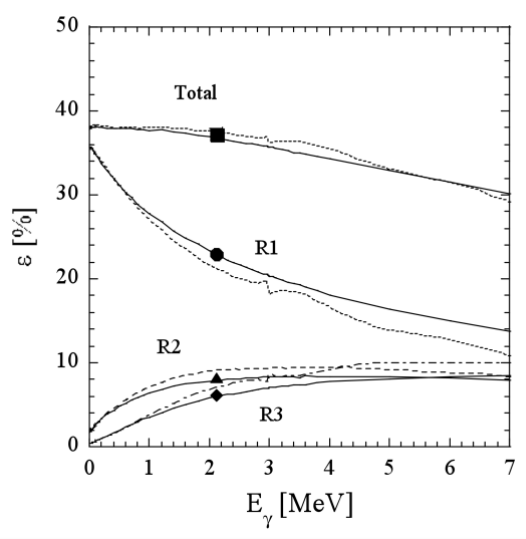

Fig. 4 Total and partial detection efficiencies of the flatresponse neutron detector for neutron-evaporation spectra (solid lines) and monochromatic neutrons (dotted lines).

The neutron angular distribution effect on the detection efficiency was investigated for isotropic s- and anisotropic p-wave neutrons with respect to the linear polarization of the laser Compton-scattering $\gamma$-ray beam. It was confirmed that the FRD has nearly identical efficiencies for s- and p-wave neutrons, where the strong spatial anisotropy in the p-wave neutron emission is smeared out during the thermalization of neutrons in the moderator. This is a common feature of moderationbased neutron detectors of the Livermore type.

Figure 5 shows distributions of the arrival-time of neutrons at the FRD for single, double, and triple neutron events in the ${ }^{209} \mathrm{Bi}(\gamma, \mathrm{xn})$ reaction at $34 \mathrm{MeV}$. Time distributions of the $1^{\text {st }}$ - and $2^{\text {nd }}$-arriving neutrons and the $1^{\text {st }}-, 2^{\text {nd }}-$, and $3^{\text {rd }}$-arriving neutrons are also shown for the double and triple neutron events, respectively. It was found that the sum distribution is 
best fitted with a linear combination of two exponential functions with time constants, $\sim 10 \mu \mathrm{s}$ for the fast component and $\sim 100 \mu \mathrm{s}$ for the slow component, respectively. One can see that the major part of the $1^{\text {st }}$ arriving neutrons is well characterized by the fast component, while the $2^{\text {nd }}$ and $3^{\text {rd }}$ components by the slow component. The fast component may represent neutron detection with the first ring located at $5.5 \mathrm{~cm}$, while the slow component neutron detection with the second and third rings at 13 and $16 \mathrm{~cm}$. The arrival-time distribution is a good subject to investigate by a Monte Carlo simulation.
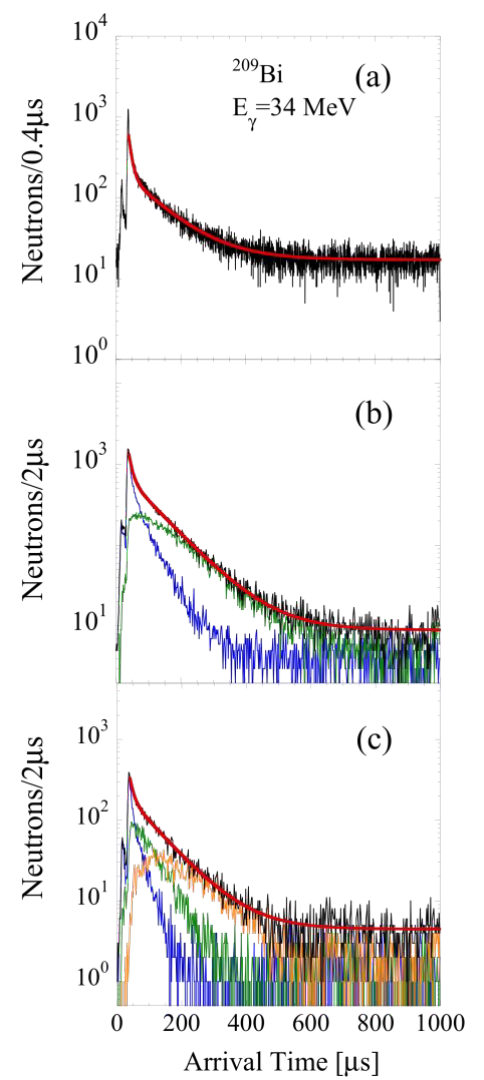

Fig. 5 (color online) Arrival-time distributions of neutrons detected with the flat-response detector for single (a), double (b), and triple (c) neutron events in the ${ }^{209} \mathrm{Bi}(\gamma, \mathrm{xn})$ reactions at $34 \mathrm{MeV}$. For the double neutron events, the arrival-time distribution is shown for the $1^{\text {st }}$ (blue) and $2^{\text {nd }}$-arriving (green) neutrons as well as the sum neutrons. Similarly, for the triple neutron events, the arrival-time distribution is shown for the $1^{\text {st }}$ - (blue), $2^{\text {nd }}$ - (green), and $3^{\text {rd }}$-arriving (orange) neutrons as well as the sum neutrons. The best fits to the single (a), double sum (b), and triple sum events (c) are shown by the red lines.

\section{New ${ }^{209} \mathrm{Bi}(\gamma, \mathrm{xn})$ data}

The technique of the direct neutron-multiplicity sorting was first used to measure ${ }^{209} \mathrm{Bi}(\gamma, \mathrm{xn})$ cross sections [13] in the IAEA-CRP. Figure 6 shows partial photoneutron cross sections obtained in the new measurement in comparison with the TALYS [14] calculation of the giant-dipole resonance (GDR) and quasi-deuteron component $[15,16]$. The GDR cross section was obtained within the Hartree-Fock-Bogolyubov plus quasiparticle random phase approximation approach on the basis of the D1M Gogny interaction both for the E1 [17] and M1 [18] components. See Ref. [13] for details.

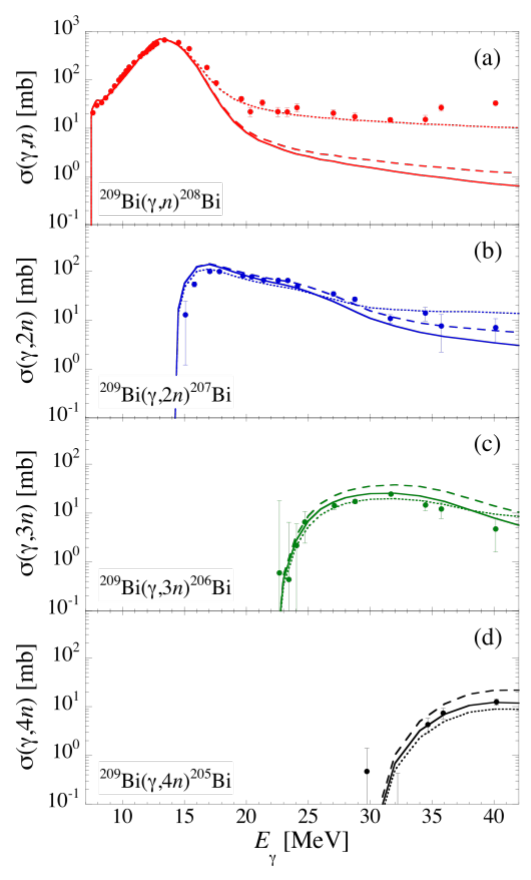

Fig. 6 (color online) Comparison with the present partial photoneutron cross sections with the TALYS calculation with the default preequilibrium parametrization (solid lines), the modified Levinger parameter $L=20$ with (dotted lines) and without (dashed lines) surface effects $\left(\mathrm{E}_{\text {surf }}=1\right.$ $\mathrm{MeV})$.

\subsection{Puzzle of the ${ }^{209} \mathrm{Bi}(\gamma, 1 \mathrm{n})$ cross section}

While the $(\gamma, \mathrm{xn})$ cross sections with $\mathrm{x}=2,3$, and 4 are reasonably well reproduced by the TALYS calculation with the default preequilibrium parametrization (solid lines), the large $(\gamma, 1 \mathrm{n})$ cross section above $25 \mathrm{MeV}$ is highly puzzling. The standard statistical model predicts small $(\gamma, 1 \mathrm{n})$ cross sections $(3-4 \mathrm{mb})$ in this energy range. Furthermore, it is kinematically considered that kineticenergies of neutrons emitted in the $(\gamma, 1 \mathrm{n})$ channel are very large; they are outside the energy range $(0.01-5.0$ 
$\mathrm{MeV})$ in which the flat-response is assured.

Although the $(\gamma, 1 \mathrm{n})$ cross section can be artificially enhanced by tuning the Levinger parameter involved in the quasi-deuteron model or even by tuning the statistical model parameters within a reasonable range, the large $(\gamma, 1 \mathrm{n})$ cross section is most likely experimental; it may be caused by secondary $\gamma$-rays produced by electrons and positrons resulting from the pair production and Compton scattering of the $\gamma$-ray beam in passing through a thick $(10 \mathrm{~cm}){ }^{209} \mathrm{Bi}$ target. A simulation shows that contaminant $(\gamma, 1 \mathrm{n})$ reactions are induced by secondary $\gamma$ rays most effectively around the peak of the GDR, thus significantly reducing the relevant $(\gamma, 1 \mathrm{n})$ cross section at high energies. We experimentally investigate this effect by changing the thickness of ${ }^{209} \mathrm{Bi}$ targets. We will write an erratum paper after a thorough investigation in experiment and simulation.

The authors thank P. von Neumann-Cosel for his comment on the neutron kinetic energy expected for the ${ }^{209} \mathrm{Bi}(\gamma, 1 \mathrm{n})$ reaction in relation to the large cross section at high energies. I.G., M.K., and D.F. acknowledge the support from the Extreme Light Infrastructure Nuclear Physics (ELI-NP) Phase II, a project co-financed by the Romanian Government and the European Union through the European Regional Development Fund - the Competitiveness Operational Programme (1/07.07.2016, COP, ID 1334). S.G. acknowledges the support from the F.R.S.-FNRS. H.U. acknowledges the support from the Premier Project of the Konan University. K.S. and S.B. acknowledge support from the Russian Foundation for Basic Research (Grant No. 15-02-05939). G.T. acknowledges the support from the Research Council of Norway under contract No. 262952. D.S. acknowledges the support of Deutsche Forschungsgemeinschaft through grant No. SFB 1245. This work was supported by the IAEA under Contracts No. ROM-20476, No. RUS-20501, and No. JPN-20564 for the CRP F41032 on Updating the Photonuclear Data Library.

\section{References}

1. http://www.lasti.u-hyogo.ac.jp/NS-en/

2. V.V. Varlamov, B.S. Ishkhanov, Phys. Part. Nucl. 35, 459 (2004).

3. V.V. Varlamov, B.S. Ishkhanov, V.N. Orlin, K.A. Stopani, Eur. Phys. J. A 50, 114 (2014).

4. A. Schiller et al., Nucl. Instrum. Phys. Res. A 447, 498 (2000).

5. H. K. Toft et al., Phys. Rev. C 81, 064311 (2010).

6. D.M. Filipescu et al., Phys. Rev. C 90, 064616 (2014).

7. H.-T. Nyhus et al., Phys. Rev. C 91, 015808 (2015).

8. B.L. Berman and S.C. Fultz, Rev. Mod. Phys. 47, 713 (1975).

9. https://www-nds.iaea.org/CRP-photonuclear/
10. Properties of neutron sources, IAEA-TECDOC410 IAEA (1987) March.

11. V. Weisskopf, Phys. Rev. 52 (1937) 295.

12. T. Kawano, Private communication.

13. I. Gheorghe et al., Phy. Rev. C 96, 044604 (2017).

14. A. J. Koning and D. Rochman, Nucl. Data Sheets 113, 2841 (2012).

15. M. L. Terranova, D. A. De Lima, and J. D. Pinheiro Filho, Europhys. Lett. 9, 523 (1989).

16. M. B. Chadwick, P. Oblozinsky, P. E. Hodgson, and G. Reffo, Phys. Rev. C 44, 814 (1991).

17. M. Martini, S. Peru, S. Hilaire, S. Goriely, and F. Lechaftois, Phys. Rev. C 94, 014304 (2016).

18. V. Tselyaev, N. Lyutorovich, J. Speth, S. Krewald, and P. G. Reinhard, Phys. Rev. C 94, 034306 (2016). 